\title{
An Application of Association Rules Discovery to Geographic Information Systems
}

\author{
Ansaf Salleb and Christel Vrain \\ LIFO - Université d'Orléans - BP 6759 \\ 45067 Orléans Cedex 2 - France \\ $\{$ salleb,cv\}@lifo.univ-orleans.fr
}

\begin{abstract}
In this paper, we are interested in the problem of extracting spatial association rules in Geographic Information Systems (GIS). We propose an algorithm that extends existing methods to deal with spatial and non-spatial data over multiple layers. It handles hierarchical, multi-valued attributes, and produces general spatial association rules. We also present a prototype, which has been applied on a real and large geographic database in the field of mineral exploration.
\end{abstract}

\section{Introduction}

In this paper, we are interested in Spatial Data Mining [46] practical applications in many domains, such as geographic information systems (GIS). We present an application of mining association rules between geographic layers according to spatial and non-spatial properties of the objects.

The concept of association rules introduced by Agrawal [1] has been extended by Koperski and Han [7] to Spatial Data. For instance, the rule:

$I s(X$, largetown $) \wedge$ Intersect $(X$, highway $) \rightarrow \operatorname{AdjacentTo}(X$, water $)(86 \%)$

expresses that $86 \%$ of the large towns intersecting highways are also adjacent to water areas (rivers, lakes, ... ).

They have proposed an algorithm to discover such rules. In their works, nonspatial information is organized into hierarchies, and rules including this information are learned. Nevertheless, information belonging to different levels of hierarchies cannot appear in a same rule. We have extended their framework to introduce non-spatial predicates in rules, even when attributes are not hierarchical, and we have applied it to a real application provided by BRGM1 1 .

The application we are interested in is explained in Section 2 and formalized in Section 3. In Section 4, we present an algorithm for extracting spatial association rules in GIS and a prototype that has been developed and applied on geographic data provided by BRGM. We conclude with a discussion and perspectives, in Section 6.

\footnotetext{
1 BRGM ('Bureau des Recherches Géologiques et Minières') is a French public institution, present in all regions of France and in some 40 countries abroad. Based on the Earth Sciences, its expertise relates to mineral resources, pollution, risks, and the management of geological environment (http://www.brgm.fr).
}

D.A. Zighed, J. Komorowski, and J. Żytkow (Eds.): PKDD 2000, LNAI 1910, pp. 613-618, 2000 (C) Springer-Verlag Berlin Heidelberg 2000 


\section{The Application}

A GIS stores data in two distinct parts: spatial data representing an object by its geometric shape (a point, a line or a polygon) and its location on the map, and non-spatial data storing properties of the geographic objects. Moreover, a GIS uses the notion of thematic layers in order to separate data into classes of same kinds. Each thematic layer is described by a relational table where each tuple is linked to a geometric object. For instance, the GIS Andes developed by BRGM [2] is a homogeneous information system of the entire Andes Cordillera. There are different layers in the system, which can be combined in any way by the user (Figure 1). Our goal is to find association rules between a given thematic layer and others layers, according to spatial relations and non-spatial attributes.

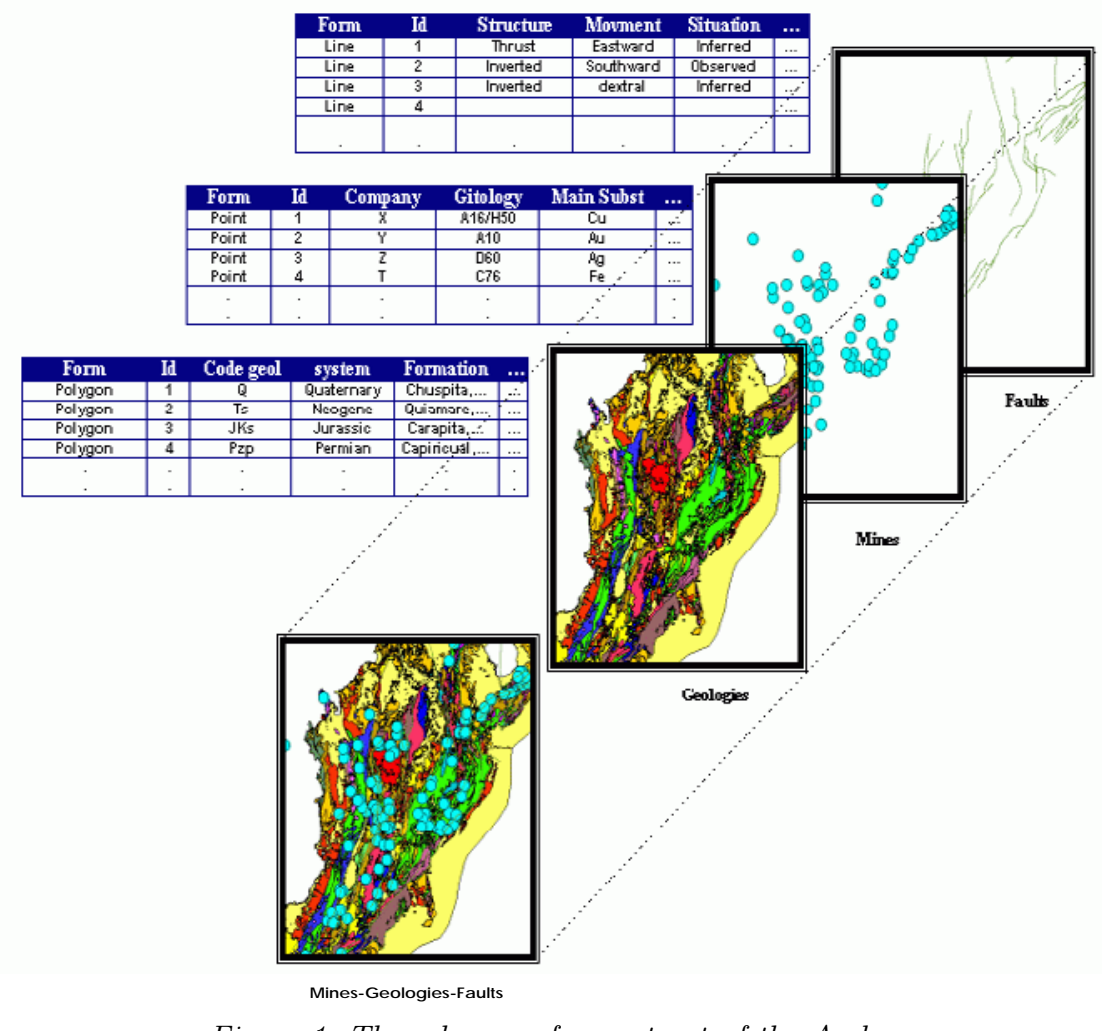

Figure 1. Three layers of an extract of the Andes

\section{Problem Formalization}

We define two kinds of layers: the Reference Layer is the thematic layer which contains the spatial objects we focus on. It is unique, and in the following, the variable $x$ is associated to the Reference Layer. A Descriptive Layer contains objects that have spatial relations with those of the reference layer. 
In our work, a spatial association rule is a rule like:

$$
P_{1}, P_{2}, \ldots, P_{n} \rightarrow Q_{1}, Q_{2}, \ldots, Q_{m}
$$

where $P_{i}, Q_{j}$, are predicates. A predicate may be:

1. a definition predicate: It defines a thematic layer, its syntax is:

Definition_Predicate(var). For instance, in the example of Section 2, Mine(x) is a definition predicate for the layer Mines.

2. a spatial predicate: It links the reference layer with a descriptive layer. Its general syntax is: Spatial_Predicate $\left(\right.$ var $\left._{i}, v \operatorname{vr}_{j}\right)$.

For instance, Near_to $(x, z)$ denotes the proximity between $x$ and $z$.

3. a non-spatial predicate: It describes a layer, its general syntax is: NonSpatial_Predicate (var,Value). In our example, Main_Substance(x, Ag) expresses that the mine $x$ has silver as main substance.

Our formalism allows to express non-spatial properties on objects. The algorithm below is able to discover association rules defined as follows:

- A non spatial association rule is a rule composed of the definition predicate concerning the reference layer and non-spatial predicates.

- A spatial association rule is a rule composed of at least two definition predicates, at least a spatial predicate and at least a non-spatial predicate.

\section{The System}

\section{General Algorithm}

Inputs:

- a spatial database $S D B$, a relational database $R D B$, taxonomies $T D B$.

- a reference Layer: $R l$

- descriptive Layers: $D l=\left\{L_{i} / i=1, \ldots, n\right\}$

- a set of non-spatial attributes for $R l$ and for each $L_{i} \in D l$

- a set of spatial predicates to compute: $S P$

- Buffer, MinSupp, MinConf

Outputs: solid, multi-levels, spatial and non-spatial association rules. Begin

Step 1: Creation of link tables: $L D B=\bigcup_{i=1}^{n} L D B_{i}$

For each $L_{i} \in D l$

- search Spatial Relations between $R l$ and $L_{i}$

- update $L D B_{i}$

Step 2: Computation of frequent predicate sets

- Creation of sets of predicates

- For each example $E_{j}$ of $L D B$

- Search the predicates sets that are true for $E_{j}$ and increase their support

- Keep predicate sets that have sufficient support

Step 3: Generation of strong Spatial Association Rules

End

Details of the Algorithm

Let us notice first that each spatial object in a given layer has a unique key or 
identifier. The algorithm is based on the building of link tables, each one relating the reference layer to a descriptive layer. The structure of a link table is composed of the following fields: the reference layer key, non-spatial attributes of the reference layer, a spatial relation linking an object of the reference layer and an object of the descriptive layer, the descriptive layer key, and non-spatial attributes of the descriptive layer. An example $E_{j}$ is a set of tuples, belonging to different link tables, that have the same reference layer key $j[3]$.

The support of a rule represents the percent of examples verifying all the predicates of the rule at the same time. A rule holds with confidence $c \%$, if $c \%$ of the examples of the Reference layer verifying the predicates given in the condition of the rule, also satisfy the predicates of the consequence of the rule.

Step 1 creates a link table $L D B_{i}$ between the reference layer $R l$ and each descriptive layer of $D l$, by searching for each object $O_{j}$ of $R l$, and for each layer of $D l$, the objects $O$ verifying at least a spatial predicate $\operatorname{sp}\left(O_{j}, O\right)$. Then, frequent predicate sets are computed. For each link table $L D B_{i}$, a conjunction composed of a single predicate is created, as follows:

- Each non-spatial attribute of $R l$ (resp. $L_{i}$ in the $L D B_{i}$ ) becomes a non-spatial binary predicate with variable $x$ (resp. $y_{i}$ ).

- The set of constants that can appear in the second argument of the predicates are computed as follows:

- For each non-hierarchical attribute, we extract in the $L D B_{i}$ its set of values.

- For each hierarchical attribute, values are obtained from its taxonomy.

- To each possible value of an attribute corresponds a predicate with this value as constant (second argument of the predicate, see Section 3).

- Each variable $y_{i}$ must be linked to $x$ by a spatial predicate (because the support concerns only the reference layer).

- The support of each predicate in the $L D B_{i}$ is computed, and frequent predicates are kept in a list $L$.

- As in apriori, predicate sets of size $\mathrm{k}$ are generated by combination of frequent predicate sets of size (k-1), and only those with a sufficient support are kept. Step 3 is a classical process for generating association rules from the set $L$.

Example: Let us suppose that we specify in the inputs the following parameters: - Reference layer $R l=$ Mines, with non-spatial attributes: $\{$ Gitology, Main_Subst $\}$. Note that Gitology is a hierarchical attribute (it takes its possibles values from the nodes of its taxonomy: $\left.A_{1}, A_{2}, A_{3}, \ldots\right)$.

- Descriptive layer $L_{1}=$ Geologies, with attributes: $\{$ System, Code_Geol $\}$.

- $S p=\{$ Included_in, Near_to $\}$, Buffer $=10 \mathrm{~km}$, MinSupp $=5 \%$ and MinConf $=40 \%$ We aim at finding relations between Mines deposits, represented by points, and the nearest Geologies (polygons).

The link table $L D B_{1}$ is constructed, as shown in Figure 2. For instance, the example $E_{j}$ is composed of two tuples of key=2 in the link table and means that the mine number 2 is included in Geology 102 and near to Geology 3.

The support table of large predicate sets is then built. Note here that the predicate sets with frequency less than 130 (which represents $5 \%$ of 2618 mines in the $R l$ ) are filtered out (Figure 3). 


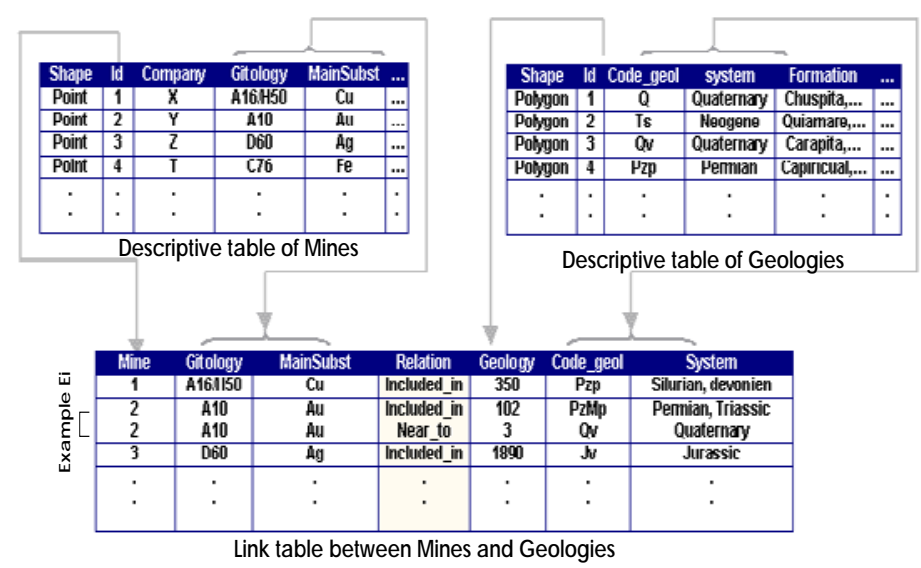

Figure 2. Link table of the example

\begin{tabular}{|c|c|c|}
\hline Num & Frequent Predicate Sets & Frequency \\
\hline 1 & Gitology $(x, A)$ & $\mathbf{5 3 3}$ \\
\hline 2 & Gitology $(x, A 1)$ & 491 \\
\hline 3 & Main_substance $(x, \bar{A} g)$ & 529 \\
\hline 4 & Main_substance $(x, A u)$ & 459 \\
\hline 5 & Geologic_Code $(y, P z s)$, Is_inside $(x, y)$ & 384 \\
\hline 6 & System (y, Ordovician), Is_inside $(x, y)$ & 214 \\
\hline 7 & Gitology $(x, A)$, Gitology $(x, A 1)$ & 491 \\
\hline 8 & Gitology $(x, A 1)$, Main_substance $(x, A(g)$ & 180 \\
\hline 9 & Gitology $(\mathrm{x}, \mathrm{A} 1)$, Geologic_Code $(\mathrm{y}, \mathrm{PZS})$, Is_inside $(\mathrm{x}, \mathrm{y})$ & 177 \\
\hline 10 & Geologic_Code (y, Pzs), System (y, Ordovician), Is inside(x,y) & 209 \\
\hline
\end{tabular}

Figure 3. An extract of the support table

Based on the support table, the algorithm generates the following rule with a support of $6.67 \%$ :

$$
\begin{aligned}
\operatorname{Mine}(x) & \wedge \operatorname{Geology}(y) \wedge \text { Code_Geol }(y, P z s) \wedge \text { Included_In }(x, y) \\
& \rightarrow \operatorname{Gitology}(x, A 1) \quad(6.67 \%, 46.09 \%)
\end{aligned}
$$

which expresses that $46.09 \%$ of mines included in geologies of code $P z s$ have as gitology $A 1$. We can classify generated association rules into three kinds:

- Statistic rules: they give the repartition of items in a hierarchy, such as:

$$
\operatorname{Mine}(x) \wedge \operatorname{Gitolog} y(x, A) \rightarrow \operatorname{Gitology}(x, A 1) \quad(92.12 \%)
$$

- Control rules: experts can also verify some known correlations:

$$
\text { Mine }(x) \wedge \operatorname{Gitology}(x, H 12) \rightarrow \text { MainSubstance }(x, A U) \quad(89.32 \%)
$$

- New rules: as for instance, the following rule with confidence $43.75 \%$ :

Mine $(x) \wedge$ Fault $(z) \wedge$ Gitology $(x, C 5) \wedge N e a r \_t o(x, z) \rightarrow$ Structure $(z$, Strike_slip $)$

We have implemented the algorithm presented in this paper, as a research prototype, named ARGIS. It has been developed in Avenue(c), an object oriented language available in ArcViewC2. In ARGIS, the user can formulate the inputs by means of an interactive graphical user interface. ARGIS handles multi-valued attributes and taxonomies, and the user can choose the levels that interest him.

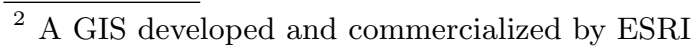


We have experimented the prototype on 3 layers of GIS Andes, a database of about 150 mega bytes (15 MB for the Mines layer, $130 \mathrm{MB}$ for the Geology layer and $5 \mathrm{MB}$ for the Fault layer) composed of about 23 thousands of records, each time using a reference layer and a descriptive layer. The process has discovered about 70 spatial association rules, and some have been considered as very interesting by the experts.

\section{Discussion and Conclusion}

The system presented in this paper is an extension of a previous work of Koperski and Han [7, and an application of mining spatial association rules in GIS. First we have added non-spatial predicates to spatial ones. Second, as suggested by Koperski in [5], we can mine rules at cross-levels of taxonomies. However, to get interesting rules at low levels of the hierarchies, the support must be low. This leads to a lot of rules because we cannot give a variable support threshold according to the level in the hierarchies as done by Koperski, whose method is guided by a scroll of all the hierarchies at the same time. In order to handle a large number of layers, and in view of the phenomenal growth of data, the efficiency of the system must be improved in the following directions: pruning useless and redundant rules, interaction with statistics, dealing with numeric data, parallelization and use of multidimensional trees.

Acknowledgments: We wish to thank the BRGM, especially the Geology and Metallogeny Research group for providing us the opportunity to experiment our algorithm on their databases. This work is supported by BRGM and by 'Région Centre'.

\section{References}

1. R. Agrawal and R. Srikant. Fast Algorithms for Mining Association Rules in Large Databases. In Proc. 1994 Int. Conf. VLDB, pages 487-499, 1994.

2. D. Cassard. Gis andes: A metallogenic gis of the andes cordillera. In 4th Int. Symp. on Andean Geodynamics, pages 147-150. IRD Paris, October 1999.

3. L. Dehaspe and L. De Raedt. Mining association rules in multiple relations. In Proc. of the 7th Int. Workshop on ILP. Springer, 1997.

4. M. Ester, H.-P. Kriegel, and J. Sander. Spatial data mining: A database approach. Lecture Notes in Computer Science, 1262:47-66, 1997.

5. K. Koperski. A progressive Refinement Approach To Spatial Data Mining. PhD thesis, Computing Science, Simon Fraser University, 1999.

6. K. Koperski, J. Adhikary, and J. Han. Spatial data mining: Progress and challenges. In SIGMOD'96 Workshop.DMKD'96, Canada, June 1996.

7. K. Koperski and J. Han. Discovery of spatial association rules in geographic information databases. Lecture Notes In Computer Science, 951:47-66, 1995.

8. J. F. Roddick and M. Spiliopoulou. A bibliography of temporal, spatial and spatiotemporal data mining research. In SIGKDD Explorations, June 1999. 